\title{
Transverse Vibration and Waves in a Membrane: Frequency Domain Spectral Element Modeling and Analysis
}

\author{
Jungho Park, Ilwook Park, and Usik Lee \\ Department of Mechanical Engineering, Inha University, 100 Inharo, Nam-gu, Incheon 402-751, Republic of Korea \\ Correspondence should be addressed to Usik Lee; ulee@inha.ac.kr
}

Received 23 June 2014; Revised 12 August 2014; Accepted 13 August 2014; Published 9 September 2014

Academic Editor: Gen Qi Xu

Copyright (C) 2014 Jungho Park et al. This is an open access article distributed under the Creative Commons Attribution License, which permits unrestricted use, distribution, and reproduction in any medium, provided the original work is properly cited.

\begin{abstract}
Although the spectral element method (SEM) has been well recognized as an exact continuum element method, its application has been limited mostly to one-dimensional (1D) structures, or plates that can be transformed into 1D-like problems by assuming the displacements in one direction of the plate in terms of known functions. We propose a spectral element model for the transverse vibration of a finite membrane subjected to arbitrary boundary conditions. The proposed model is developed by using the boundary splitting method and the waveguide FEM-based spectral super element method in combination. The performance of the proposed spectral element model is numerically validated by comparison with exact solutions and solutions using the standard finite element method (FEM).
\end{abstract}

\section{Introduction}

A membrane is a two-dimensional (2D) structure that can be characterized by the dominating tension and its negligible resistance to bending. The dynamic analysis of membrane has been an important research subject because of its applications to various engineering devices such as loudspeakers, microphones, membrane valves, pressure regulators, and antennae for space communications [1-3].

Exact solutions are well known for the free vibration of a membrane with simple geometry such as rectangle, circle, or ellipse [4-6]. However, no closed-form solutions exist for membranes with complex geometry. Thus, various solution techniques have been developed by many researchers: they include the finite difference method $[7,8]$, the superposition method using wave-type base functions [9], the discrete singular convolution method [10, 11], the Kantorovich method [12], the optimized Galerkin-Kantorovich method [13], the hybrid method composed of differential transforms and the Kantorovich method [14], the differential quadrature method $[13,15]$, the collocation approach based on the little sinc functions [16], the Rayleigh-Ritz method [17], and the finite element method (FEM) [18-24].

The FEM is one of most widely used computational methods that can be applied to various engineering problems.
The interpolation functions used for the FEM are independent of vibrating frequency. The accuracy of the FEM depends on the size of the finite elements, which should be less than one-eighth of the wave length. Accordingly, a huge number of finite elements must be used to improve the accuracy of the FEM, especially in a high frequency regime. This can result in a significant increase in computation cost. In contrast to the FEM, the spectral element method (SEM) is a frequency domain element method that can provide accurate dynamic responses at very high frequencies [25]. The shape (or interpolation) functions used in the SEM are derived from exact solutions of the governing equations of motion. Thus, only one element is sufficient to obtain the exact dynamic behavior of a uniform structural member at any frequency, which can significantly reduce the computation cost and time.

Despite the advantages of the SEM, its application has been limited to one-dimensional structures, the Levy-type plates [26], and the plates that are infinite in one direction [27]. To the authors' knowledge, the SEM has not been applied to 2D structures such as membranes and plates with arbitrary boundary conditions.

The purpose of this study is to develop a spectral element model for the transverse vibration of a membrane by using 
the boundary splitting method and the waveguide FEMbased spectral super element method (SSEM) [28]. The high accuracy of the proposed spectral element model is evaluated by comparison with exact theoretical solutions and solutions based on the standard FEM.

\section{Formulation of the Spectral Element Model}

The equation of motion for the transverse vibration of a membrane is given by

$$
P\left(\frac{\partial^{2} w}{\partial x^{2}}+\frac{\partial^{2} w}{\partial y^{2}}\right)-\rho \frac{\partial^{2} w}{\partial t^{2}}=f(x, y, t),
$$

where $w(x, y, t)$ is the transverse displacement, $f(x, y, t)$ is the external force applied normal to the surface of the membrane, $P$ is a constant tensile force, and $\rho$ is the mass per unit surface area of the membrane.

The first step in the spectral element formulation is to transform the time domain governing equation into the frequency domain by representing the dynamic response $w(x, y, t)$ and external force $f(x, y, t)$ in spectral forms, as follows:

$$
\begin{aligned}
& \{w(x, y, t), f(x, y, t)\} \\
& \quad=\frac{1}{M} \sum_{m=0}^{M-1}\left\{\bar{w}_{m}(x, y), \bar{f}_{m}(x, y)\right\} e^{i \omega_{m} t},
\end{aligned}
$$

where $i=\sqrt{-1}$ is the imaginary unitand $M$ is the number of samples for the fast Fourier transform- (FFT-) based spectral analysis [29]. The quantities with overbars denote the spectral components of the corresponding time domain quantities, and the subscripts $m$ are omitted in the following derivation for the sake of brevity. Substituting (2) into (1) gives the following governing equation in the frequency domain:

$$
P\left(\frac{\partial^{2} \bar{w}}{\partial x^{2}}+\frac{\partial^{2} \bar{w}}{\partial y^{2}}\right)+\rho \omega^{2} \bar{w}=\bar{f}(x, y) .
$$

Next, we obtain free wave solutions for a finite membrane element subjected to arbitrary boundary conditions. To that end, we consider the homogeneous governing equation that can be reduced from (3) by letting $\bar{f}(x, y)=0$ as follows:

$$
P\left(\frac{\partial^{2} \bar{w}}{\partial x^{2}}+\frac{\partial^{2} \bar{w}}{\partial y^{2}}\right)+\rho \omega^{2} \bar{w}=0 .
$$

By using the boundary splitting method [30], the solution of (4) is obtained by summing the solutions of two partial problems subjected to different boundary conditions, as shown in Figure 1:

$$
\bar{w}(x, y)=\bar{w}_{A}(x, y)+\bar{w}_{B}(x, y),
$$

where $\bar{w}_{A}(x, y)$ is the solution when the finite membrane element has null (or fixed) boundary conditions at two opposite edges at $y=-L_{y} / 2$ and $L_{y} / 2$ and $\bar{w}_{B}(x, y)$ is the solution when the finite membrane element has null boundary conditions at two opposite edges at $x=-L_{x} / 2$ and $L_{x} / 2$.
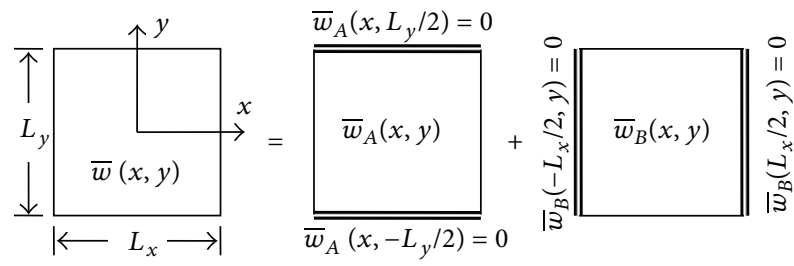

FIGURE 1: Splitting boundary conditions to obtain the dynamic response $\bar{w}(x, y)$ of a membrane subjected to arbitrary boundary conditions.

In this study, $\bar{w}_{A}(x, y)$ and $\bar{w}_{B}(x, y)$ were obtained by using the concept of SSEM introduced in [28]. In the SSEM, a rectangular problem domain is considered to be the assembly of a number of rectangular strip elements in one direction (say, in the $y$-direction). Similar to the FEM, the displacement fields in a rectangular strip element (of width $l_{y}$ in the $y$ direction) are then represented in terms of the nodal degrees of freedom (DOFs) defined on two node lines at $y=0$ and $y=l_{y}$ by using polynomial-based shape functions in the $y$-direction. The difference between this approach and the FEM is that the nodal DOFs are defined as functions of $x$ and are then analytically determined in the frequencywavenumber domain by using the waveguide FEM approach. The approaches to obtaining $\bar{w}_{A}(x, y)$ and $\bar{w}_{B}(x, y)$ will be addressed in detail in the following.

The weak form of (4) can be written as follows:

$$
\begin{aligned}
\int_{x} \int_{y}\{ & P \frac{\partial \bar{w}}{\partial x} \delta\left(\frac{\partial \bar{w}}{\partial x}\right) \\
& \left.+P \frac{\partial \bar{w}}{\partial y} \delta\left(\frac{\partial \bar{w}}{\partial y}\right)-\rho \omega^{2} \bar{w} \delta \bar{w}\right\} d x d y=0 .
\end{aligned}
$$

2.1. Derivation of $\bar{w}_{A}(x, y)$. Consider a finite membrane element that is fixed at two opposite edges at $y=-L_{y} / 2$ and $L_{y} / 2$ but is subjected to arbitrary boundary conditions at the other two opposite edges. The finite membrane element is divided into $N_{y}$ strips in the $y$-direction as shown in Figure 2(a). The details of the eth strip element are shown in Figure 2(b). In Figure 2 and in subsequent figures, dots "•" denote active nodes at which the transverse displacements will be determined.

The displacement field in the eth strip element can be represented by

$$
\bar{w}_{A}^{(e)}(x, y)=\mathbf{Y}_{A}^{(e)}(y) \mathbf{v}_{A}^{(e)}(x) \quad\left(y_{e-1} \leq y \leq y_{e}\right),
$$

where

$$
\begin{aligned}
\mathbf{Y}_{A}^{(e)}(y) & =\left[Y_{1}^{(e)}(y), Y_{2}^{(e)}(y)\right] \\
& =\left[-l_{y}^{(e)-1}\left(y-y_{e}\right), l_{y}^{(e)-1}\left(y-y_{e-1}\right)\right], \\
\mathbf{v}_{A}^{(e)}(x) & =\left\{\bar{W}_{A}^{(e-1)}(x), \bar{W}_{A}^{(e)}(x)\right\}^{T}
\end{aligned}
$$




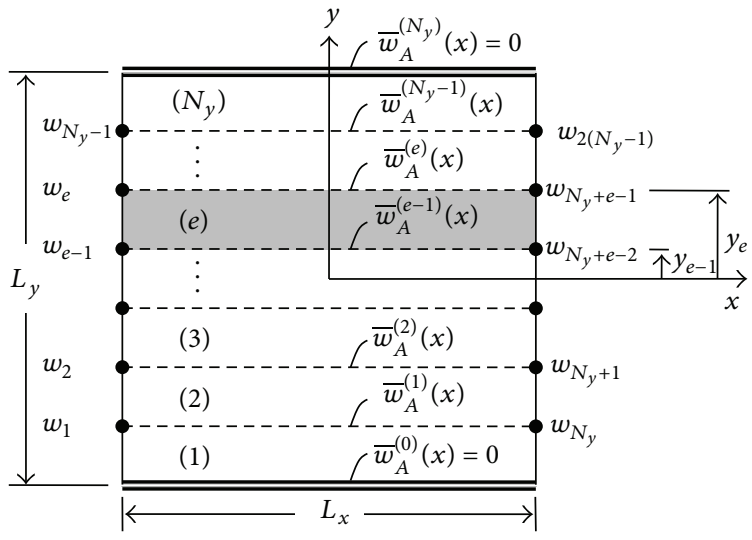

(a)

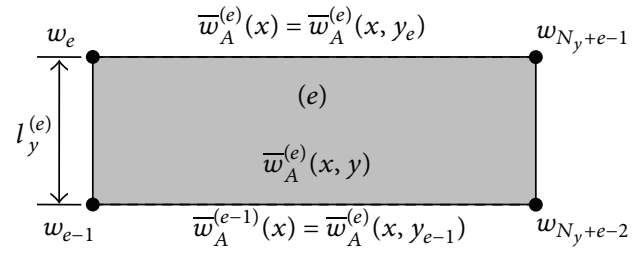

(b)

FIGURE 2: Finite strip element representation of a membrane subjected to null boundary conditions at $y=-L_{y} / 2$ and $L_{y} / 2$ to obtain the solution $\bar{w}_{A}(x, y): \bullet=$ active nodes.

with the following definitions:

$$
\begin{gathered}
\bar{W}_{A}^{(e-1)}(x)=\bar{w}_{A}^{(e)}\left(x, y_{e-1}\right), \\
\bar{W}_{A}^{(e)}(x)=\bar{w}_{A}^{(e)}\left(x, y_{e}\right) .
\end{gathered}
$$

By using (7), the displacement field over the whole domain of the membrane can be represented as

$$
\bar{w}_{A}(x, y)=\mathbf{Y}_{A}(y) \mathbf{v}_{A}(x),
$$

where

$$
\begin{aligned}
\mathbf{Y}_{A}(y)= & h_{A}^{(1)} Y_{2}^{(1)}+h_{A}^{(2)} Y_{1}^{(2)}, h_{A}^{(2)} Y_{2}^{(2)}+h_{A}^{(3)} Y_{1}^{(3)}, \ldots, \\
& h_{A}^{(e-1)} Y_{2}^{(e-1)}+h_{A}^{(e)} Y_{1}^{(e)}, \\
& h_{A}^{(e)} Y_{2}^{(e)}+h_{A}^{(e+1)} Y_{1}^{(e+1)}, \ldots, \\
& h_{A}^{\left(N_{y}-2\right)} Y_{2}^{\left(N_{y}-2\right)}+h_{A}^{\left(N_{y}-1\right)} Y_{1}^{\left(N_{y}-1\right)}, \\
& \left.h_{A}^{\left(N_{y}-1\right)} Y_{2}^{\left(N_{y}-1\right)}+h_{A}^{\left(N_{y}\right)} Y_{1}^{\left(N_{y}\right)}\right], \\
\mathbf{v}_{A}(x)=\{ & \bar{W}_{A}^{(1)}(x), \bar{W}_{A}^{(2)}(x), \ldots, \bar{W}_{A}^{(e-1)}(x), \\
& \left.\bar{W}_{A}^{(e)}(x), \ldots, \bar{W}_{A}^{\left(N_{y}-2\right)}(x), \bar{W}_{A}^{\left(N_{y}-1\right)}(x)\right\}^{T} .
\end{aligned}
$$

In (11), $h_{A}^{(e)}(y)\left(e=1,2,3, \ldots, N_{y}\right)$ are functions defined by

$$
h_{A}^{(e)}(y)=H\left(y-y_{e-1}\right)-H\left(y-y_{e}\right),
$$

where $H(y)$ is the Heaviside unit step function. Although the displacement field representations given by (7) for each strip element are similar to those used for the SSEM in [28], the displacement field representation over the whole domain of a finite membrane element is newly developed in this study.
The null (fixed) boundary conditions at $y=-L_{y} / 2$ and $L_{y} / 2$ (i.e., $\bar{W}_{A}^{(0)}(x)=0$ and $\bar{W}_{A}^{\left(N_{y}\right)}(x)=0$ ) have been applied to (10).

By substituting (10) into (6), we obtain

$$
\mathbf{A}_{A 2} \frac{\partial^{2} \mathbf{v}_{A}(x)}{\partial x^{2}}+\mathbf{A}_{A 1} \mathbf{v}_{A}(x)-\omega^{2} \mathbf{M}_{A} \mathbf{v}_{A}(x)=0,
$$

where

$$
\begin{aligned}
& \mathbf{A}_{A 1}=P \int_{-L_{y} / 2}^{L_{y} / 2} \frac{\partial \mathbf{Y}_{A}^{T}}{\partial y} \frac{\partial \mathbf{Y}_{A}}{\partial y} d y=P l_{y}^{-1} \mathbf{C}_{1\left(N_{y}\right)}, \\
& \mathbf{A}_{A 2}=-P \int_{-L_{y} / 2}^{L_{y} / 2} \mathbf{Y}_{A}^{T} \mathbf{Y}_{A} d y=-\frac{1}{6} P l_{y} \mathbf{C}_{2\left(N_{y}\right)}, \\
& \mathbf{M}_{A}=\rho \int_{-L_{y} / 2}^{L_{y} / 2} \mathbf{Y}_{A}^{T} \mathbf{Y}_{A} d y=\frac{1}{6} \rho l_{y} \mathbf{C}_{2\left(N_{y}\right)} .
\end{aligned}
$$

The constant matrices $\mathbf{C}_{1(q)}$ and $\mathbf{C}_{2(q)}$ are provided in Appendix A for the case in which the membrane is divided into $q$ identical strips (i.e., $l_{y}=l_{y}^{(e)}=L_{y} / q$ ).

Assume a solution to (14) in the following form:

$$
\begin{aligned}
\mathbf{v}_{A}(x) & =\left\{\begin{array}{c}
1 \\
r_{2} \\
\vdots \\
r_{N_{y}-1}
\end{array}\right\} a e^{-i\left(k_{x} x+(1 / 2) \bar{k}_{x} L_{x}\right)} \\
& =\mathbf{r} a e^{-i\left(k_{x} x+(1 / 2) \bar{k}_{x} L_{x}\right)},
\end{aligned}
$$

where $a$ is constant, $k_{x}$ is the wavenumber in the $x$-direction, and

$$
\bar{k}_{x}= \begin{cases}-k_{x} & \text { if } \operatorname{Im}\left(k_{x}\right)>0 \\ +k_{x} & \text { if } \operatorname{Im}\left(k_{x}\right)<0 \\ 0 & \text { if } \operatorname{Im}\left(k_{x}\right)=0\end{cases}
$$


Substituting (16) into (14) gives the following eigenvalue problem:

$$
\left[-k_{x}^{2} \mathbf{A}_{A 2}+\mathbf{A}_{A 1}-\omega^{2} \mathbf{M}_{A}\right] \mathbf{r}_{A}=0 .
$$

The dispersion relation (frequency-wavenumber relationship) can be obtained from (18) as follows:

$$
\operatorname{det}\left[-k_{x}^{2} \mathbf{A}_{A 2}+\mathbf{A}_{A 1}-\omega^{2} \mathbf{M}_{A}\right]=0 .
$$

By using the wavenumbers $k_{x(j)}\left(j=1,2,3, \ldots, 2\left(N_{y}-1\right)\right)$ computed from (19), the solution of (14) can be written in the form

$$
\mathbf{v}_{A}(x)=\mathbf{R}_{A} \mathbf{E}_{A}(x ; \omega) \mathbf{a},
$$

where

$$
\begin{aligned}
& \mathbf{a}=\left\{\begin{array}{lllllll}
a_{1} & a_{2} & a_{3} & \cdots & a_{i} & \cdots & a_{2\left(N_{y}-1\right)}
\end{array}\right\}^{T}, \\
& \mathbf{R}_{A}=\left[\begin{array}{lllllll}
\mathbf{r}_{A(1)} & \mathbf{r}_{A(2)} & \mathbf{r}_{A(3)} & \cdots & \mathbf{r}_{A(j)} & \cdots & \mathbf{r}_{A\left(2\left(N_{y}-1\right)\right)}
\end{array}\right], \\
& \mathbf{E}_{A}(x ; \omega)=\text { diagonal }\left[e^{-i\left[k_{x(j)} x+(1 / 2) \bar{k}_{x(j)} L_{x}\right]}\right], \\
& \left(j=1,2,3, \ldots, 2\left(N_{y}-1\right)\right) \text {. }
\end{aligned}
$$

In (21), $\mathbf{r}_{A(j)}$ is the wave mode vector, which can be determined from (18) for the $j$ th wavenumber $k_{x(j)}$.

The nodal DOFs at $x=-L_{x} / 2$ and $L_{x} / 2$ can be written in vector form as

$$
\mathbf{d}_{A}=\left\{\begin{array}{l}
\mathbf{d}_{A 1} \\
\mathbf{d}_{A 2}
\end{array}\right\}
$$

where

$$
\begin{aligned}
\mathbf{d}_{A 1}= & \mathbf{v}_{A}\left(-\frac{L_{x}}{2}\right) \\
= & \left\{W_{1}, W_{2}, \ldots, W_{e-1}, W_{e}, \ldots, W_{N_{y}-2}, W_{N_{y}-1}\right\}^{T} \\
\mathbf{d}_{A 2}= & \mathbf{v}_{A}\left(+\frac{L_{x}}{2}\right) \\
= & \left\{W_{N_{y}}, W_{N_{y}+1}, \ldots, W_{N_{y}+e-2}, W_{N_{y}+e-1}, \ldots,\right. \\
& \left.W_{2\left(N_{y}-1\right)-1}, W_{2\left(N_{y}-1\right)}\right\}^{T} .
\end{aligned}
$$

By using (20), the nodal DOFs vector $\mathbf{d}_{A}$ can be written in terms of the constant vector a as follows:

$$
\mathbf{d}_{A}=\left[\begin{array}{l}
\mathbf{R}_{A} \mathbf{E}_{A}\left(-\frac{L_{x}}{2}\right) \\
\mathbf{R}_{A} \mathbf{E}_{A}\left(+\frac{L_{x}}{2}\right)
\end{array}\right] \mathbf{a}=\mathbf{H}_{A}(\omega) \mathbf{a} .
$$

The constant vector a can be removed from (20) by using (24), which yields

$$
\mathbf{v}_{A}(x)=\mathbf{X}_{A}(x ; \omega) \mathbf{d}_{A},
$$

where

$$
\mathbf{X}_{A}(x ; \omega)=\mathbf{R}_{A} \mathbf{E}_{A}(x ; \omega) \mathbf{H}_{A}(\omega)^{-1}
$$

By substituting (25) into (10), the solution $\bar{w}_{A}(x, y)$ can be obtained in the form as follows:

$$
\bar{w}_{A}(x, y)=\mathbf{Y}_{A}(y) \mathbf{X}_{A}(x ; \omega) \mathbf{d}_{A}=\mathbf{N}_{A}(x, y ; \omega) \mathbf{d}_{A} .
$$

2.2. Derivation of $\bar{w}_{B}(x, y)$. For the solution $\bar{w}_{B}(x, y)$, consider the same finite membrane element that was considered in Section 2.1. However, the element is now assumed to be fixed at two opposite edges at $x=-L_{x} / 2$ and $L_{x} / 2$ but subjected to arbitrary boundary conditions at the other two opposite edges. The finite membrane element is divided into $N_{x}$ strips in the $x$-direction as shown in Figure 3(a) and the details of the eth strip element are shown in Figure 3(b).

The solution procedure to obtain $\bar{w}_{B}(x, y)$ is identical to that used to obtain $\bar{w}_{A}(x, y)$ in Section 2.1, except for the change of coordinate directions. Thus, without repeating the same solution procedure, the solution $\bar{w}_{B}(x, y)$ can be written as follows:

$$
\bar{w}_{B}(x, y)=\mathbf{X}_{B}(x) \mathbf{Y}_{B}(y ; \omega) \mathbf{d}_{B}=\mathbf{N}_{B}(x, y ; \omega) \mathbf{d}_{B},
$$

where

$$
\begin{aligned}
& \mathbf{d}_{B}=\left\{W_{1}, W_{2}, \ldots, W_{e-1}, W_{e}, \ldots, W_{N_{x}-2},\right. \\
& W_{N_{x}-1}, W_{N_{x}}, W_{N_{x}+1}, \ldots, W_{N_{x}+e-2},
\end{aligned}
$$

$$
\begin{gathered}
\left.W_{N_{x}+e-1}, \ldots, W_{2\left(N_{x}-1\right)-1}, W_{2\left(N_{x}-1\right)}\right\}^{T} \\
\mathbf{X}_{B}(x)=\left[h_{B}^{(1)} X_{2}^{(1)}+h_{B}^{(2)} X_{1}^{(2)}, h_{B}^{(2)} X_{2}^{(2)}+h_{B}^{(3)} X_{1}^{(3)}, \ldots,\right. \\
h_{B}^{(e-1)} X_{2}^{(e-1)}+h_{B}^{(e)} X_{1}^{(e)}, h_{B}^{(e)} X_{2}^{(e)}+h_{B}^{(e+1)} X_{1}^{(e+1)} \\
\ldots, h_{B}^{\left(N_{x}-2\right)} X_{2}^{\left(N_{y}-2\right)}+h_{B}^{\left(N_{x}-1\right)} X_{1}^{\left(N_{x}-1\right)}, \\
\left.h_{B}^{\left(N_{x}-1\right)} X_{2}^{\left(N_{x}-1\right)}+h_{B}^{\left(N_{x}\right)} X_{1}^{\left(N_{x}\right)}\right] \\
\mathbf{Y}_{B}(y ; \omega)=\mathbf{R}_{B} \mathbf{E}_{B}(y ; \omega) \mathbf{H}_{B}(\omega)^{-1} .
\end{gathered}
$$

In (30), the following definitions are used:

$$
\begin{gathered}
h_{B}^{(e)}(x)=H\left(x-x_{e-1}\right)-H\left(x-x_{e}\right), \\
X_{1}^{(e)}(y)=-l_{x}^{(e)-1}\left(x-x_{e}\right), \\
X_{2}^{(e)}(y)=l_{x}^{(e)-1}\left(x-x_{e-1}\right),
\end{gathered}
$$




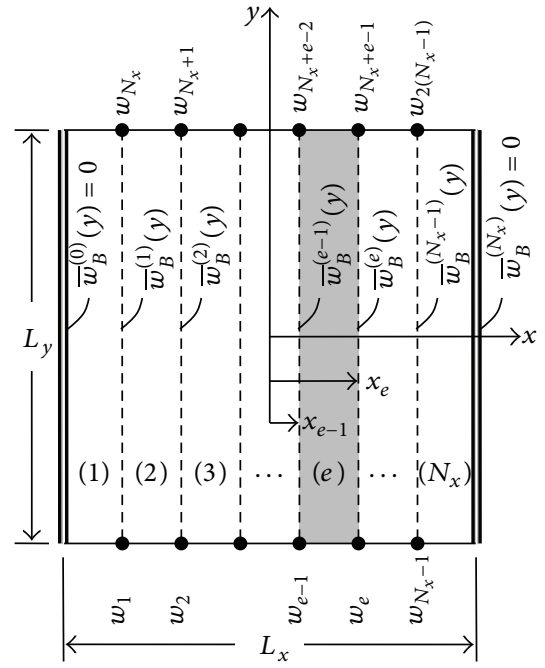

(a)

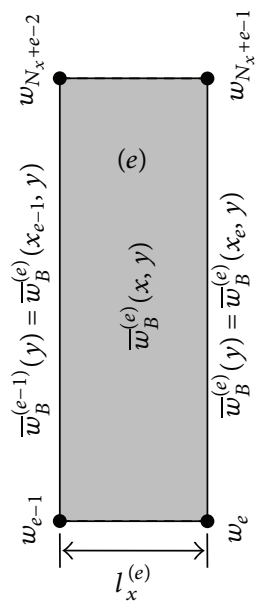

(b)

FIGURE 3: Finite strip element representation of a membrane subjected to null boundary conditions at $x=-L_{x} / 2$ and $L_{x} / 2$ to obtain the solution $\bar{w}_{B}(x, y): \bullet=$ active nodes.

$$
\begin{aligned}
& \mathbf{R}_{B}=\left[\begin{array}{lllllll}
\mathbf{r}_{B(1)} & \mathbf{r}_{B(2)} & \mathbf{r}_{B(3)} & \cdots & \mathbf{r}_{B(j)} & \cdots & \mathbf{r}_{B\left(2\left(N_{x}-1\right)\right)}
\end{array}\right], \\
& \mathbf{E}_{B}(y ; \omega)=\operatorname{diagonal}\left[e^{-i\left[k_{y(j)} y+(1 / 2) \bar{k}_{y(j)} L_{y}\right]}\right], \\
& \left(j=1,2,3, \ldots, 2\left(N_{x}-1\right)\right), \\
& \mathbf{H}_{B}(\omega)=\left[\begin{array}{l}
\mathbf{R}_{B} \mathbf{E}_{B}\left(-\frac{L_{y}}{2}\right) \\
\mathbf{R}_{B} \mathbf{E}_{B}\left(+\frac{L_{y}}{2}\right)
\end{array}\right],
\end{aligned}
$$

where

$$
\bar{k}_{y(j)}= \begin{cases}-k_{y(j)} & \text { if } \operatorname{Im}\left(k_{y(j)}\right)>0 \\ +k_{y(j)} & \text { if } \operatorname{Im}\left(k_{y(j)}\right)<0 \\ 0 & \text { if } \operatorname{Im}\left(k_{y(j)}\right)=0\end{cases}
$$

Notice that $k_{y(j)}$ and $\mathbf{r}_{B(j)}$ are the $j$ th wavenumber and wave mode vector, respectively, satisfying the following eigenvalue problem at a frequency $\omega$ :

$$
\left[-k_{y(j)}^{2} \mathbf{A}_{B 2}+\mathbf{A}_{B 1}-\omega^{2} \mathbf{M}_{B}\right] \mathbf{r}_{B(j)}=0
$$

where

$$
\begin{aligned}
& \mathbf{A}_{B 1}=P l_{x}^{-1} \mathbf{C}_{1\left(N_{x}\right)}, \\
& \mathbf{A}_{B 2}=-\frac{1}{6} P l_{x} \mathbf{C}_{2\left(N_{x}\right)}, \\
& \mathbf{M}_{B}=\frac{1}{6} \rho l_{x} \mathbf{C}_{2\left(N_{x}\right)} .
\end{aligned}
$$

2.3. Derivation of $\bar{w}(x, y)$. The full displacement field $\bar{w}(x, y)$ in a finite membrane element can be obtained by substituting (27) and (28) into (5) as follows:

$$
\begin{aligned}
\bar{w}(x, y) & =\mathbf{N}_{A}(x, y ; \omega) \mathbf{d}_{A}+\mathbf{N}_{B}(x, y ; \omega) \mathbf{d}_{B} \\
& =\mathbf{N}(x, y ; \omega) \mathbf{d},
\end{aligned}
$$

where $\mathbf{N}(x, y ; \omega)$ is the 1-by-2( $\left.N_{x}+N_{y}-2\right)$ dynamic shape function matrix defined by

$$
\mathbf{N}(x, y ; \omega)=\left[\mathbf{N}_{A}(x, y ; \omega) \mathbf{N}_{B}(x, y ; \omega)\right]
$$

and $\mathbf{d}(\omega)$ is the $2\left(N_{x}+N_{y}-2\right)$-by-1 spectral nodal DOFs vector defined by

$$
\mathbf{d}=\left\{\begin{array}{l}
\mathbf{d}_{A} \\
\mathbf{d}_{B}
\end{array}\right\} .
$$

2.4. Formulation of Spectral Element Equation. The weak form of the governing equation of forced vibration (3) can be derived in the following form:

$$
\begin{aligned}
& \int_{x} \int_{y}\left\{P \frac{\partial \bar{w}}{\partial x} \delta\left(\frac{\partial \bar{w}}{\partial x}\right)\right. \\
& \left.+P \frac{\partial \bar{w}}{\partial y} \delta\left(\frac{\partial \bar{w}}{\partial y}\right)-\rho \omega^{2} \bar{w} \delta \bar{w}\right\} \\
& =\int_{x} \int_{y} \bar{f}(x, y) \delta \bar{w} d x d y \\
& +\int_{y} Q_{x 1}(y) \delta \bar{w}\left(-\frac{L_{x}}{2}, y\right) d y \\
& +\int_{y} Q_{x 2}(y) \delta \bar{w}\left(\frac{L_{x}}{2}, y\right) d y
\end{aligned}
$$$$
\left.+P \frac{\partial \bar{w}}{\partial y} \delta\left(\frac{\partial \bar{w}}{\partial y}\right)-\rho \omega^{2} \bar{w} \delta \bar{w}\right\} d x d y
$$ 


$$
\begin{aligned}
& +\int_{x} Q_{y 1}(x) \delta \bar{w}\left(x,-\frac{L_{y}}{2}\right) d x \\
& +\int_{x} Q_{y 2}(x) \delta \bar{w}\left(x, \frac{L_{y}}{2}\right) d x
\end{aligned}
$$

where $Q_{x 1}(y), Q_{x 2}(y), Q_{y 1}(x)$, and $Q_{y 2}(x)$ are external forces applied on four boundary edges. Substituting (35) into (38) gives

$$
\begin{aligned}
\delta \mathbf{d}^{T}\left[\int_{x} \int_{y}\right. & \left(P \frac{\partial \mathbf{N}^{T}}{\partial x} \frac{\partial \mathbf{N}}{\partial x}+P \frac{\partial \mathbf{N}^{T}}{\partial y} \frac{\partial \mathbf{N}}{\partial y}\right. \\
& \left.\left.-\omega^{2} \rho \mathbf{N}^{T} \mathbf{N}\right) d x d y\right] \mathbf{d}(\omega) \\
=\delta \mathbf{d}^{T}\left[\int_{x} \int_{y} \bar{f}(x, y) \mathbf{N}^{T} d x d y\right. & \\
& +\int_{y} Q_{x 1}(y) \mathbf{N}^{T}\left(-\frac{L_{x}}{2}, y\right) d y \\
& +\int_{y} Q_{x 2}(y) \mathbf{N}^{T}\left(\frac{L_{x}}{2}, y\right) d y \\
& +\int_{x} Q_{y 1}(x) \mathbf{N}^{T}\left(x,-\frac{L_{y}}{2}\right) d x \\
& \left.+\int_{x} Q_{y 2}(x) \mathbf{N}^{T}\left(x, \frac{L_{y}}{2}\right) d x\right] .
\end{aligned}
$$

Since $\delta \mathbf{d}$ is the arbitrary variation of $\mathbf{d}(\omega)$, the spectral element equation can be obtained from (39) as follows:

$$
\mathbf{S}(\omega) \mathbf{d}(\omega)=\mathbf{f}_{1}(\omega)+\mathbf{f}_{2}(\omega),
$$

where $\mathbf{S}(\omega)$ is the $2\left(N_{x}+N_{y}-2\right)$-by- $2\left(N_{x}+N_{y}-2\right)$ dynamic stiffness matrix (or spectral element matrix) defined by

$$
\begin{aligned}
\mathbf{S}(\omega) & =\int_{x} \int_{y}\left(P \frac{\partial \mathbf{N}^{T}}{\partial x} \frac{\partial \mathbf{N}}{\partial x}+P \frac{\partial \mathbf{N}^{T}}{\partial y} \frac{\partial \mathbf{N}}{\partial y}-\omega^{2} \rho \mathbf{N}^{T} \mathbf{N}\right) d x d y \\
& =\mathbf{H}^{-T}(\omega)\left[\mathbf{D}_{x}(\omega)+\mathbf{D}_{y}(\omega)-\omega^{2} \mathbf{D}_{\rho}(\omega)\right] \mathbf{H}^{-1}(\omega)
\end{aligned}
$$

and $\mathbf{f}_{1}(\omega)$ and $\mathbf{f}_{2}(\omega)$ are the spectral nodal force vectors defined by

$$
\begin{aligned}
\mathbf{f}_{1}(\omega)= & \int_{x} \int_{y} \bar{f}(x, y) \mathbf{N}^{T}(x, y) d x d y \\
\mathbf{f}_{2}(\omega)= & \int_{y} Q_{x 1}(y) \mathbf{N}^{T}\left(-\frac{L_{x}}{2}, y\right) d y \\
& +\int_{y} Q_{x 2}(y) \mathbf{N}^{T}\left(\frac{L_{x}}{2}, y\right) d y \\
& +\int_{x} Q_{y 1}(x) \mathbf{N}^{T}\left(x,-\frac{L_{y}}{2}\right) d x \\
& +\int_{x} Q_{y 2}(x) \mathbf{N}^{T}\left(x, \frac{L_{y}}{2}\right) d x .
\end{aligned}
$$

In (41), $\mathbf{H}(\omega)$ is the $2\left(N_{x}+N_{y}-2\right)$-by- $2\left(N_{x}+N_{y}-2\right)$ matrix defined by

$$
\mathbf{H}(\omega)=\left[\begin{array}{cc}
\mathbf{H}_{A}(\omega) & \mathbf{0} \\
\mathbf{0} & \mathbf{H}_{B}(\omega)
\end{array}\right]
$$

and the matrices $\mathbf{D}_{x}, \mathbf{D}_{y}$, and $\mathbf{D}_{\rho}$ are defined in Appendix $\mathrm{B}$.

\section{Numerical Results and Discussion}

3.1. Spectral Element (SE) Analysis. Once the spectral element model represented by (40) has been derived, the spectral element analysis for a problem is straight forward. The spectral element analysis procedure is very similar to that used in the standard FEM [25]. The whole domain of the problem can be discretized into a number of finite elements, and the spectral element equations for each finite element are assembled in a manner analogous to that of the FEM. After imposing relevant boundary conditions, the governing equation of the global dynamic system can be obtained in the form

$$
\mathbf{S}_{g}(\omega) \mathbf{d}_{g}=\mathbf{f}_{1 g}+\mathbf{f}_{2 g}=\mathbf{f}_{g},
$$

where the subscript $g$ denotes quantities of the global dynamic system.

Natural frequencies $\omega_{\mathrm{NAT}}$ of the problem can be obtained from the condition that the determinant of $\mathbf{S}_{g}(\omega)$ must vanish at $\omega_{\mathrm{NAT}}$ as follows $[25,31]$ :

$$
\operatorname{det} \mathbf{S}_{g}\left(\omega_{\mathrm{NAT}}\right)=0 \text {. }
$$

The mode shapes at natural frequencies $\omega_{\mathrm{NAT}}$ can be obtained as follows. (1) Firstly, consider homogeneous global dynamic system equation $\mathbf{S}_{g}(\omega) \mathbf{d}_{g}=\mathbf{0}$ obtained from (44) by putting $\mathbf{f}_{g}=\mathbf{0}$. (2) Secondly, compute $\mathbf{d}_{g}$ at $\omega=\omega_{\mathrm{NAT}}$ from $\mathbf{S}_{g}(\omega) \mathbf{d}_{g}=$ $\mathbf{0}$ by setting one component (usually the first component) of $\mathbf{d}_{g}$ at unit value. (3) Lastly, apply computed $\mathbf{d}_{g}$ to (35) to obtain the mode shapes.

From (44), the spectral nodal DOFs can be computed as

$$
\mathbf{d}_{g}=\mathbf{S}_{g}(\omega)^{-1} \mathbf{f}_{g} .
$$

The dynamic responses in the frequency domain, or the frequency response functions, can be readily obtained from the system transfer matrix $\mathbf{S}_{g}(\omega)^{-1}$. The dynamic responses in the time domain can be efficiently computed from (35) where the spectral nodal DOFs vector $\mathbf{d}(\omega)$ is determined from (46) by using the inverse-FFT algorithm. A disadvantage of SEM, as compared to the standard FEM that is discussed in the next subsection, is that the global dynamic stiffness matrix $\mathbf{S}_{g}(\omega)$ and spectral nodal DOFs $\mathbf{d}_{g}$ must be computed repeatedly at all discrete frequencies up to Nyquist frequency.

3.2. Finite Element (FE) Analysis. In this study, exact solutions and FEM solutions are used to validate the proposed spectral element model. The finite element model used in this study was derived as follows:

$$
\mathbf{M} \ddot{\mathbf{d}}+\mathbf{K d}=\mathbf{f}(t),
$$




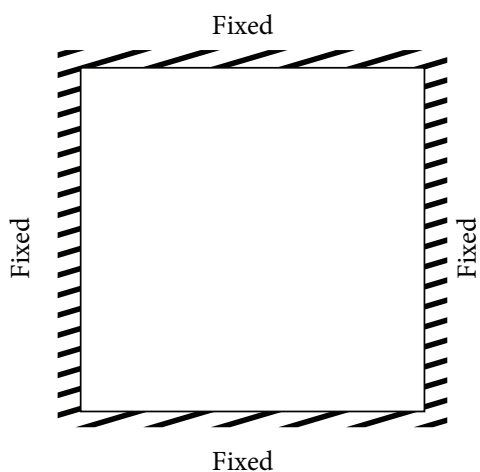

(a) Example 1

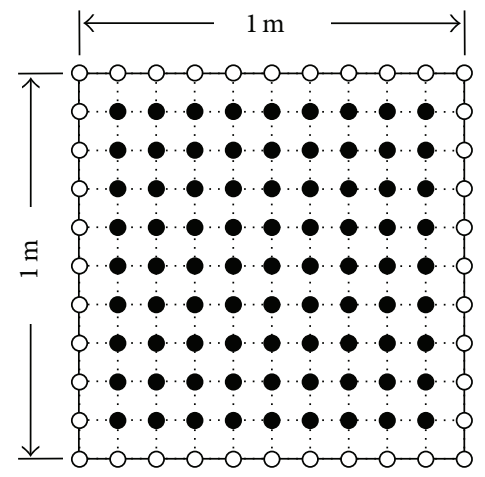

(b) FEM model

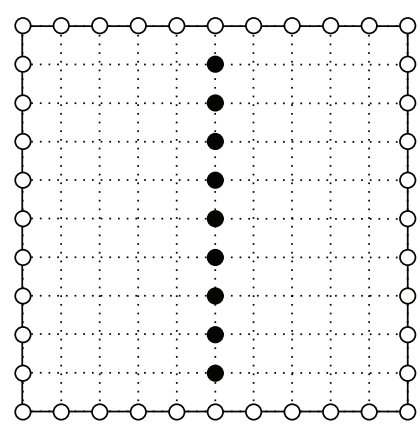

(c) SEM model

FIGURE 4: Example 1: square membrane I and its FEM and SEM models: $\bullet$ = active nodes; $\bigcirc=$ fixed nodes.

where $\mathbf{M}$ and $\mathbf{K}$ are 4-by-4 finite element mass and stiffness matrices, respectively, and are given in Appendix C. For the finite element equation (47) formulated from (1) by using a variational approach, the displacement in a finite membrane element is represented as

$$
w(x, y, t)=\mathbf{N}(x, y) \mathbf{d}(t),
$$

where

$$
\begin{aligned}
& \mathbf{N}(x, y)= {\left[\left(1-l_{x}^{-1} x\right)\left(1-l_{y}^{-1}\right), l_{x}^{-1}\left(1-l_{y}^{-1}\right),\right.} \\
&\left.l_{x}^{-1} l_{y}^{-1} x y,\left(1-l_{x}^{-1}\right) l_{y}^{-1}\right], \\
& \mathbf{d}(t)=\left\{w_{1}(t), w_{2}(t), w_{3}(t), w_{4}(t)\right\}^{T},
\end{aligned}
$$

where $w_{1}(t), w_{2}(t), w_{3}(t)$, and $w_{4}(t)$ are the nodal displacements specified at the four corner nodes of the finite membrane element.

3.3. Numerical Results. In our numerical results, we assumed that example membranes have identical mass densities of $\rho=2700 \mathrm{~kg} / \mathrm{m}^{3}$ and are subjected to a constant tensile force $P=1 \mathrm{kN} / \mathrm{m}$. To evaluate the proposed spectral element model by comparison with the natural frequencies obtained by the exact theory or FEM, we considered three types of membranes: two square membranes subjected to different boundary conditions and one $\Gamma$-shaped membrane.

Figure 4(a) shows the first square membrane (square membrane I). It has the dimensions of $L=L_{x}=L_{y}=$ $1 \mathrm{~m}$, and all boundaries are fixed. In Table 1 , the natural frequencies (in $\mathrm{Hz}$ ) obtained by FEM and SEM are compared with the exact frequencies given by

$$
\begin{array}{r}
f_{m n}=\frac{1}{2} \sqrt{\frac{P}{\rho}} \sqrt{\left(\frac{m}{L_{x}}\right)^{2}+\left(\frac{n}{L_{y}}\right)^{2}}(\mathrm{~Hz}), \\
(m, n=1,2,3, \ldots) .
\end{array}
$$

The FE model is illustrated in Figure 4(b) for the case in which a total of 81 nodes were used in the FE analysis.
Similarly, the SE model represented by two identical spectral elements (dimensions: $0.5 \mathrm{~m} \times 1 \mathrm{~m} ; N_{x}=5$ and $N_{y}=$ 10) is illustrated in Figure 4(c) for the case in which a total of 9 nodes were used in the SE analysis. Table 1 shows that the natural frequencies obtained by both FE and SE models converged to the exact values as the total number of (active) nodes or elements used in the analyses was increased. However, we note from Table 1 that the FEM can provide the lowest ten natural frequencies accurately (up to four digits) when more than 17,956 nodes are used, whereas the proposed SEM can accurately provide them by using only 69 nodes. The computation times required for the FE analysis using 17,956 nodes and the SE analysis using 69 nodes were about $18 \mathrm{~min}$ and $1 \mathrm{~min}$, respectively (CPU times on our laboratory PC: Intel(R) core (TM) i7-2600CPU).

Figure 5(a) shows the second square membrane (square membrane II). It has the same dimensions of $L=L_{x}=$ $L_{y}=1 \mathrm{~m}$ as that of the previous example but was subjected to different boundary conditions as shown in Figure 5(a). Table 2 shows a comparison of the natural frequencies (in $\mathrm{Hz}$ ) obtained by FEM and SEM with the exact analytical results given by

$$
\begin{array}{r}
f_{m n}=\frac{1}{2} \sqrt{\frac{P}{\rho}} \sqrt{\left(\frac{2 m-1}{2 L_{x}}\right)^{2}+\left(\frac{n}{L_{y}}\right)^{2}}(\mathrm{~Hz}), \\
(m, n=1,2,3, \ldots) .
\end{array}
$$

Figures 5(b) and 5(c) illustrate the FE model and the SE model, respectively, for the case in which a total of 90 nodes were used in the FE analysis and a total of 18 nodes in the SE analysis. Notice that the SE model is represented by two identical spectral elements (dimensions: $0.5 \mathrm{~m} \times 1 \mathrm{~m} ; N_{x}=$ 5 and $N_{y}=10$ ). Table 2 also shows that the results from both FEM and SEM converged to exact solutions as the total number of active nodes (or elements) used in the analyses was increased. More than 25,440 nodes were required for the FE analysis to accurately predict the lowest ten natural frequencies (up to four digits), and more than 258 nodes were required for the $\mathrm{SE}$ analysis. 


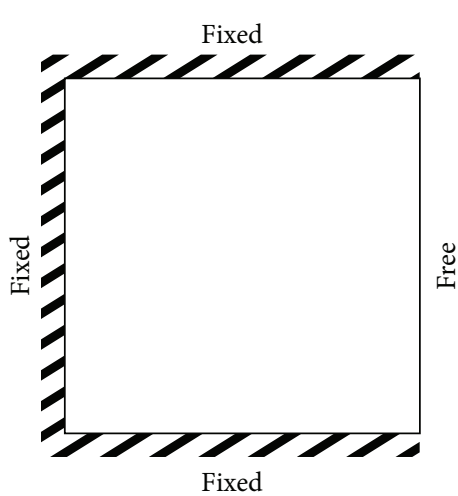

(a) Example 2

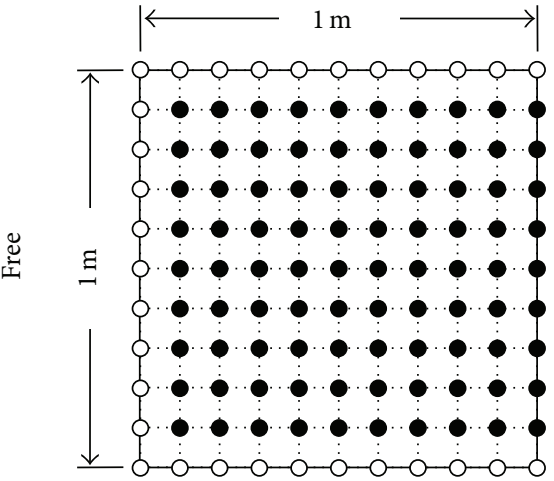

(b) FEM model

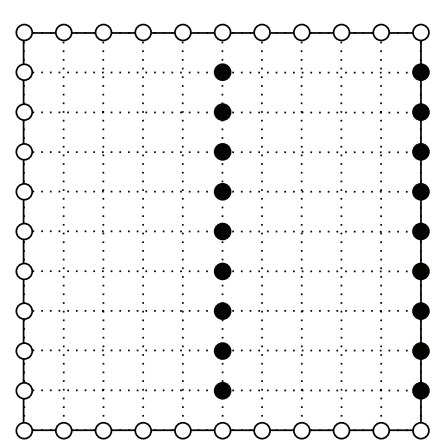

(c) SEM model

FIGURE 5: Example 2: square membrane II and its FEM and SEM models: $\bullet=$ active nodes; $\bigcirc=$ fixed nodes.

TABLE 1: Comparison of the natural frequencies $(\mathrm{Hz})$ of square membrane I obtained by exact theory, FEM, and SEM.

\begin{tabular}{lcccccccc}
\hline Modes & Exact & \multicolumn{2}{c}{ FEM } & & \multicolumn{2}{c}{ SEM } \\
& & $n_{n}=81$ & $n_{n}=2,401$ & $n_{n}=9,801$ & $n_{n}=17,956$ & $n_{n}=9$ & $n_{n}=29$ & $n_{n}=69$ \\
\hline$(1,1)$ & 0.430 & 0.432 & 0.430 & 0.430 & 0.430 & 0.431 & 0.430 \\
$(1,2)$ & 0.680 & 0.690 & 0.681 & 0.680 & 0.680 & 0.681 & 0.681 \\
$(2,2)$ & 0.861 & 0.875 & 0.861 & 0.861 & 0.861 & 0.868 & 0.862 \\
$(1,3)$ & 0.962 & 0.995 & 0.964 & 0.963 & 0.962 & 0.963 & 0.962 \\
$(2,3)$ & 1.097 & 1.131 & 1.099 & 1.098 & 1.097 & 1.102 & 1.098 \\
$(1,4)$ & 1.255 & 1.333 & 1.258 & 1.255 & 1.255 & 1.256 & 1.255 \\
$(3,3)$ & 1.291 & 1.438 & 1.293 & 1.292 & 1.291 & 1.315 & 1.294 \\
$(2,4)$ & 1.361 & 1.607 & 1.364 & 1.362 & 1.361 & 1.365 & 1.361 \\
$(3,4)$ & 1.522 & 1.705 & 1.525 & 1.522 & 1.522 & 1.542 & 1.255 \\
$(1,5)$ & 1.552 & 1.788 & 1.558 & 1.553 & 1.552 & 1.642 & 1.361 \\
\hline
\end{tabular}

Note: $n_{n}$ is the total number of active nodes used in the analysis.

TABLE 2: Comparison of the natural frequencies $(\mathrm{Hz})$ of square membrane II obtained by exact theory, FEM, and SEM.

\begin{tabular}{lcccccccc}
\hline Modes & Exact & \multicolumn{3}{c}{ FEM } & & \multicolumn{2}{c}{ SEM } \\
$n_{n}=98$ & $n_{n}=25,440$ & $n_{n}=18$ & 0.340 \\
\hline$(1,1)$ & 0.340 & 0.341 & 0.340 & 0.340 & 0.340 & 0.341 & 0.340 \\
$(2,1)$ & 0.549 & 0.553 & 0.549 & 0.549 & 0.549 & 0.549 & 0.549 \\
$(1,2)$ & 0.627 & 0.637 & 0.628 & 0.627 & 0.627 & 0.637 & 0.628 & 0.549 \\
$(2,2)$ & 0.761 & 0.771 & 0.762 & 0.761 & 0.761 & 0.769 & 0.761 \\
$(3,1)$ & 0.819 & 0.838 & 0.821 & 0.820 & 0.819 & 0.820 & 0.819 \\
$(1,3)$ & 0.926 & 0.959 & 0.929 & 0.926 & 0.926 & 0.959 & 0.927 \\
$(3,2)$ & 0.974 & 0.996 & 0.977 & 0.974 & 0.974 & 0.981 & 0.975 \\
$(2,3)$ & 1.021 & 1.053 & 1.024 & 1.021 & 1.021 & 1.051 & 0.919 \\
$(4,1)$ & 1.108 & 1.160 & 1.113 & 1.108 & 1.108 & 1.108 & 0.974 \\
$(3,3)$ & 1.188 & 1.227 & 1.193 & 1.189 & 1.188 & 1.215 & 1.108 \\
\hline
\end{tabular}

Note: $n_{n}$ is the total number of active nodes used in the analysis.

Figure 6(a) shows the $\Gamma$-shaped membrane used in our third example problem. All edges of the membrane were fixed, and the geometric dimensions are shown in Figure 6(b). Exact natural frequencies are not available in closed forms for this type of membrane. Thus, the natural frequencies from the SE analysis were compared with those from the FE analysis. Figures 6(b) and 6(c) illustrate the FE and SE models, respectively, when a total of 56 nodes were used in the FE analysis and a total of 8 nodes in the SE analysis. In the SE analysis, the membrane is represented by three identical square spectral elements (dimensions: $0.5 \mathrm{~m} \times$ $0.5 \mathrm{~m} ; N_{x}=N_{y}=5$ ). Table 3 shows the lowest ten natural frequencies sufficiently converged (up to four digits) when more than 10,561 nodes were used in the FE analysis, and 118 


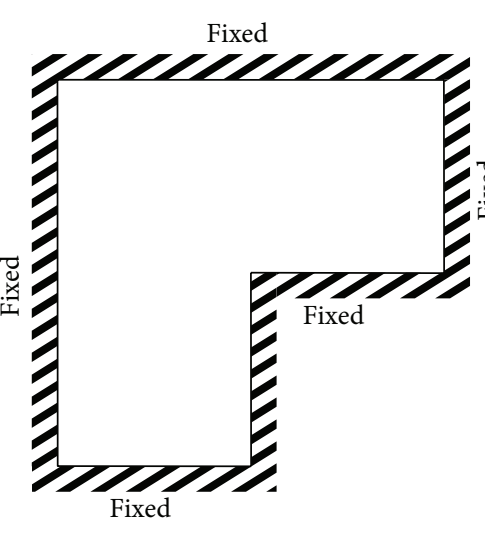

(a) Example 3

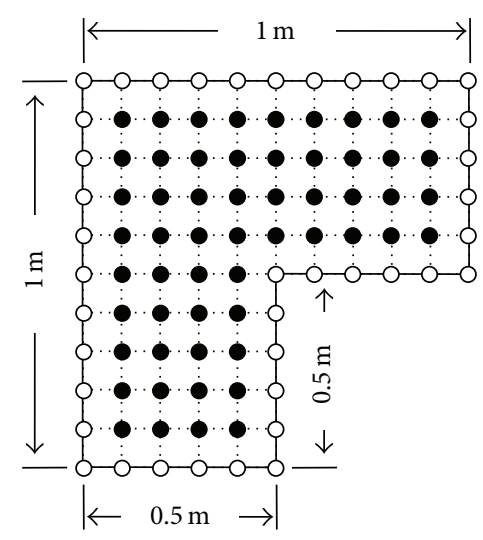

(b) FEM model

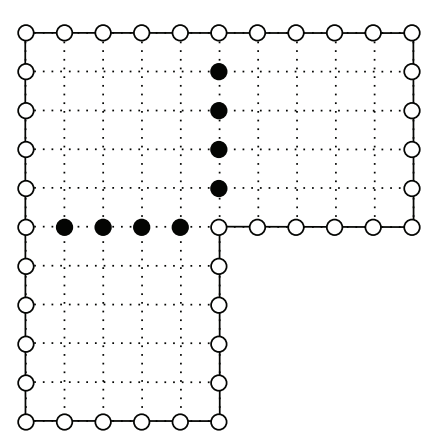

(c) SEM model

Figure 6: Example 3: $\Gamma$-type membrane and its FEM and SEM models: $\bullet=$ active nodes; $\bigcirc=$ fixed nodes.

TABLE 3: Comparison of the natural frequencies $(\mathrm{Hz})$ of the $\Gamma$-shaped membrane obtained by FEM and SEM.

\begin{tabular}{|c|c|c|c|c|c|c|c|}
\hline \multirow{2}{*}{ Modes } & \multicolumn{4}{|c|}{ FEM } & \multicolumn{3}{|c|}{ SEM } \\
\hline & $n_{n}=56$ & $n_{n}=1,776$ & $n_{n}=7,301$ & $n_{n}=10,561$ & $n_{n}=8$ & $n_{n}=48$ & $n_{n}=118$ \\
\hline 1 & 0.612 & 0.602 & 0.602 & 0.602 & 0.612 & 0.602 & 0.602 \\
\hline 2 & 0.768 & 0.756 & 0.755 & 0.755 & 0.770 & 0.756 & 0.755 \\
\hline 3 & 0.875 & 0.861 & 0.861 & 0.861 & 0.870 & 0.861 & 0.861 \\
\hline 4 & 1.086 & 1.054 & 1.053 & 1.053 & 1.061 & 1.053 & 1.053 \\
\hline 5 & 1.138 & 1.097 & 1.095 & 1.095 & 1.109 & 1.095 & 1.095 \\
\hline 6 & 1.323 & 1.251 & 1.248 & 1.248 & 1.365 & 1.251 & 1.248 \\
\hline 7 & 1.379 & 1.302 & 1.300 & 1.299 & 1.434 & 1.303 & 1.299 \\
\hline 8 & 1.438 & 1.364 & 1.362 & 1.361 & 1.527 & 1.360 & 1.361 \\
\hline 9 & 1.540 & 1.462 & 1.460 & 1.459 & 1.622 & 1.462 & 1.459 \\
\hline 10 & 1.670 & 1.571 & 1.567 & 1.567 & 1.644 & 1.570 & 1.567 \\
\hline
\end{tabular}

Note: $n_{n}$ is the total number of active nodes used in the analysis.

nodes were used in the SE analysis. The lowest nine mode shapes computed by the present SEM are shown in Figure 7.

From the aforementioned investigations, we conclude that the proposed spectral element model has the capability to provide very accurate natural frequencies and dynamic responses by using a very small number of nodes (or elements) and CPU times as compared to the standard FEM.

A point force in the form of a five-peak Morlet-wavelet input signal with a center frequency of $20 \mathrm{~Hz}$ was applied at the center of square membrane I to excite the waves propagating in the membrane. The waves propagating along the $x$ axis were predicted analytically by using the modal analysis method and numerically by using the proposed spectral element model. In the SE analysis, the square membrane was represented by two spectral elements (dimensions: $0.5 \mathrm{~m} \times$ $1 \mathrm{~m}$ ) as shown in Figure 4(c). The number of strip elements in the $x$-direction $\left(N_{x}\right)$ and the number of strip elements in the $y$-direction $\left(N_{y}\right)$ in a spectral element were increased step by step. The results, shown in Figure 8, demonstrate that the results by the SEM converged to the exact analytical solution as the values of $N_{x}$ and $N_{y}$ were increased to values larger than 250 and 500 , respectively.
Finally, Figure 9 shows the numerical simulation results of the waves propagating in square membrane I. The waves were excited by a five-peak Morlet-wavelet input point force applied at the center of the membrane. The square membrane was represented by two spectral elements (dimensions: $0.5 \mathrm{~m} \times 1 \mathrm{~m} ; N_{x}=250$ and $N_{y}=500$ ). From Figure 9, we note that the circular waves spread out until they reached the membrane edges, followed by continuing symmetric wave reflections afterward.

\section{Conclusion}

In this study, a frequency-domain spectral element model was developed for the transverse vibrations of the membranes subjected to arbitrary boundary conditions. To formulate the spectral element model, the displacement field in a finite membrane element was obtained in the frequency domain, first by splitting the boundary conditions of the finite membrane element to form two partial problems and, then, by applying the waveguide FEM-based spectral super element method to obtain displacement fields for each partial problem. The full displacement field in the finite membrane 

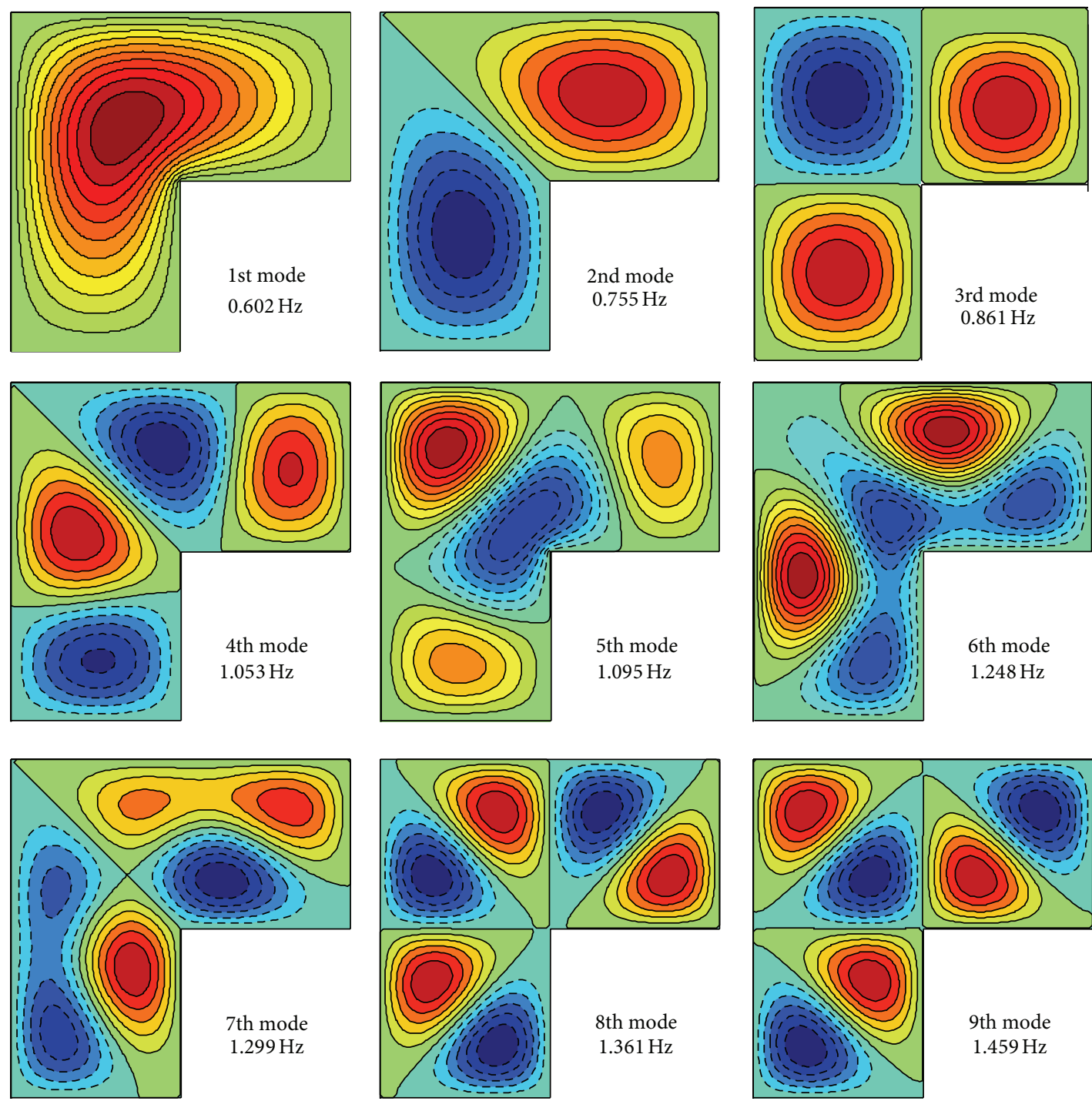

FIGURE 7: The lowest nine mode shapes for the $\Gamma$-type membrane.

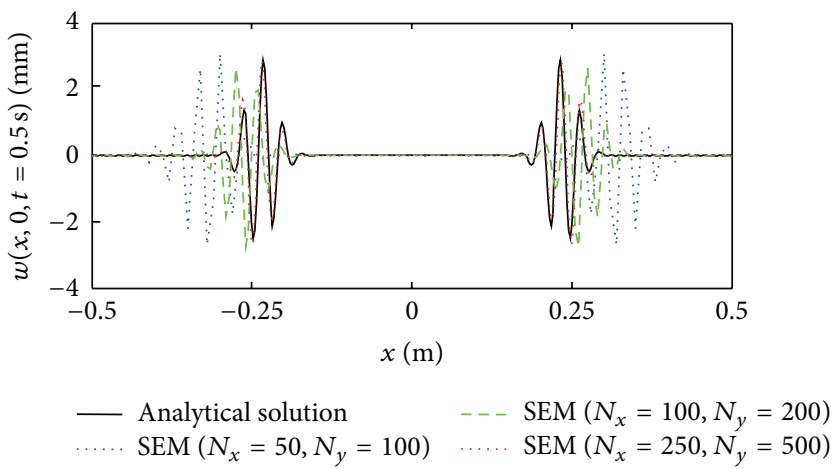

Figure 8: Waves in square membrane I excited by a point force (five-peak Morlet-wavelet input) applied at the center of the membrane.

element was then obtained by summing displacement fields obtained for two partial problems. The spectral element model formulated by using this full displacement field was validated by comparison with exact solutions and with solutions using the standard FEM. We numerically showed that the proposed spectral element model can provide very accurate natural frequencies and dynamic responses by using a relatively very small number of active nodes compared with 

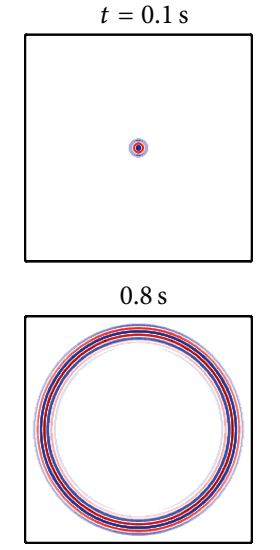

$1.2 \mathrm{~s}$

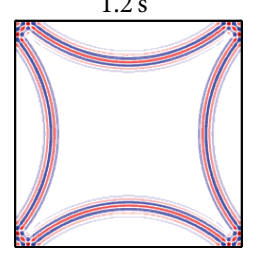

$0.2 \mathrm{~s}$

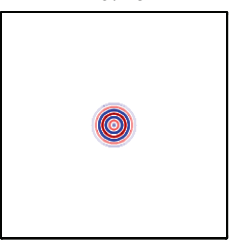

$0.9 \mathrm{~s}$

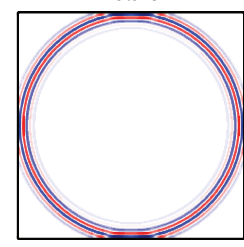

$1.3 \mathrm{~s}$

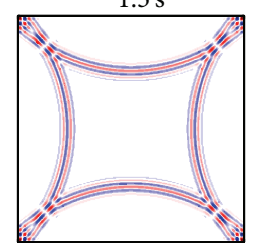

$0.4 \mathrm{~s}$

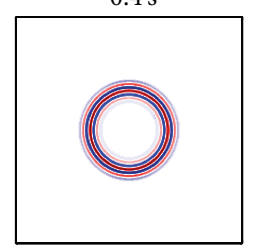

$1 \mathrm{~s}$

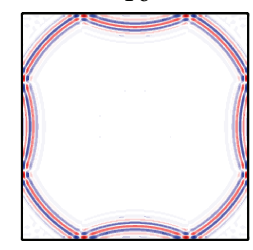

$1.4 \mathrm{~s}$

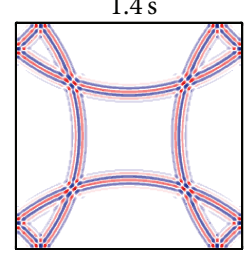

$0.6 s$

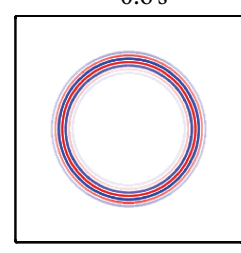

$1.1 \mathrm{~s}$

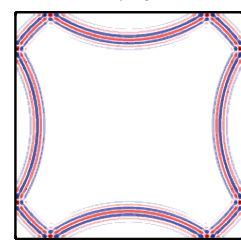

$1.5 \mathrm{~s}$

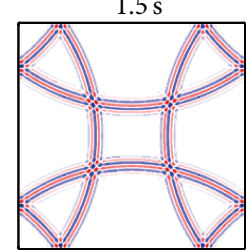

FIGURE 9: Numerical simulation of the wave propagation generated by a point five-peak Morlet-wavelet input force applied at the center of square membrane I.

the standard FEM. The spectral element formulation method presented in this study can be applied to other $2 \mathrm{D}$ structures such as plates.

\section{Appendices}

\section{A. Constant Matrices Defined in}

(15), (34), and (B.3)

Consider

$$
\begin{aligned}
\mathrm{C}_{1(q)} & =\left[\begin{array}{cccccc}
2 & -1 & 0 & \cdots & 0 & 0 \\
-1 & 2 & -1 & \cdots & 0 & 0 \\
0 & -1 & 2 & \cdots & 0 & 0 \\
\vdots & \vdots & \vdots & \ddots & -1 & 0 \\
0 & 0 & 0 & -1 & 2 & -1 \\
0 & 0 & 0 & 0 & -1 & 2
\end{array}\right]_{(q-1) \times(q-1)} \\
\mathrm{C}_{2(q)} & =\left[\begin{array}{cccccc}
4 & 1 & 0 & \cdots & 0 & 0 \\
1 & 4 & 1 & \cdots & 0 & 0 \\
0 & 1 & 4 & \cdots & 0 & 0 \\
\vdots & \vdots & \vdots & \ddots & 1 & 0 \\
0 & 0 & 0 & 1 & 4 & 1 \\
0 & 0 & 0 & 0 & 1 & 4
\end{array}\right]_{(q-1) \times(q-1)}
\end{aligned}
$$

B. Matrices $\mathbf{D}_{x}(\omega), \mathbf{D}_{y}(\omega)$, and $\mathbf{D}_{\rho}(\omega)$ in (41)

Consider

$$
\begin{aligned}
& \mathbf{D}_{x}(\omega)=P\left[\mathbf{D}_{i j}^{x}\right], \\
& \mathbf{D}_{y}(\omega)=P\left[\mathbf{D}_{i j}^{y}\right],
\end{aligned}
$$

$$
\begin{array}{r}
\mathbf{D}_{\rho}(\omega)=\rho\left[\mathbf{D}_{i j}^{\rho}\right], \\
(i, j=1,2),
\end{array}
$$

where

$$
\begin{aligned}
\mathbf{D}_{11}^{x} & =-\int_{x} \int_{y} \mathbf{E}_{A} \mathbf{K}_{A} \mathbf{R}_{A}^{T} \mathbf{Y}_{A}^{T} \mathbf{Y}_{A} \mathbf{R}_{A} \mathbf{K}_{A} \mathbf{E}_{A} d x d y \\
& =\left(-\mathbf{K}_{A} \mathbf{R}_{A}^{T} \mathbf{J}_{A A}^{Y} \mathbf{R}_{A} \mathbf{K}_{A}\right) \cdot{ }^{*} \boldsymbol{\Sigma}_{1}^{A}, \\
\mathbf{D}_{22}^{x} & =\int_{x} \int_{y} \mathbf{E}_{B} \mathbf{R}_{B}^{T} \frac{d \mathbf{X}_{B}^{T}}{d x} \frac{d \mathbf{X}_{B}}{d x} \mathbf{R}_{B} \mathbf{E}_{B} d x d y \\
& =\left(\mathbf{R}_{B}^{T} \mathbf{J}_{B B}^{X} \mathbf{R}_{B}\right) \cdot{ }^{*} \boldsymbol{\Sigma}_{1}^{B}, \\
\mathbf{D}_{12}^{x} & =\mathbf{D}_{21}^{x T}=(-i) \int_{x} \int_{y} \mathbf{E}_{A} \mathbf{K}_{A} \mathbf{R}_{A}^{T} \mathbf{Y}_{A}^{T} \frac{d \mathbf{X}_{B}}{d x} \mathbf{R}_{B} \mathbf{E}_{B} d x d y \\
& =\left(-i \mathbf{\Sigma}_{4}^{B} \mathbf{R}_{B}\right) \cdot{ }^{*}\left(\mathbf{K}_{A} \mathbf{R}_{A}^{T} \boldsymbol{\Sigma}_{3}^{A T}\right), \\
\mathbf{D}_{11}^{y} & =\int_{x} \int_{y} \mathbf{E}_{A} \mathbf{R}_{A}^{T} \frac{d \mathbf{Y}_{A}^{T}}{d y} \frac{d \mathbf{Y}_{A}}{d y} \mathbf{R}_{A} \mathbf{E}_{A} d x d y \\
& =\left(\mathbf{R}_{A}^{T} \overline{\mathbf{J}}_{A A}^{Y} \mathbf{R}_{A}\right) \cdot{ }^{*} \boldsymbol{\Sigma}_{1}^{A}, \\
\mathbf{D}_{22}^{y} & =-\int_{x} \int_{y} \mathbf{E}_{B} \mathbf{K}_{B} \mathbf{R}_{B}^{T} \mathbf{X}_{B}^{T} \mathbf{X}_{B} \mathbf{R}_{B} \mathbf{K}_{B} \mathbf{E}_{B} d x d y \\
& =\left(-\mathbf{K}_{B} \mathbf{R}_{B}^{T} \mathbf{J}_{B B}^{X} \mathbf{R}_{B} \mathbf{K}_{B}\right) \cdot{ }^{*} \boldsymbol{\Sigma}_{1}^{B},
\end{aligned}
$$




$$
\begin{aligned}
\mathbf{D}_{12}^{y} & =\mathbf{D}_{21}^{y T}=(-i) \int_{x} \int_{y} \mathbf{E}_{A} \mathbf{R}_{A}^{T} \frac{d \mathbf{Y}_{A}^{T}}{d y} \mathbf{X}_{B} \mathbf{R}_{B} \mathbf{K}_{B} \mathbf{E}_{B} d x d y \\
& =\left(-i \boldsymbol{\Sigma}_{2}^{B} \mathbf{R}_{B} \mathbf{K}_{B}\right) \cdot{ }^{*}\left(\mathbf{R}_{A}^{T} \boldsymbol{\Sigma}_{5}^{A T}\right), \\
\mathbf{D}_{11}^{\rho} & =\int_{x} \int_{y} \mathbf{E}_{A} \mathbf{R}_{A}^{T} \mathbf{Y}_{A}^{T} \mathbf{Y}_{A} \mathbf{R}_{A} \mathbf{E}_{A} d x d y \\
& =\left(\mathbf{R}_{A}^{T} \mathbf{J}_{A A}^{Y} \mathbf{R}_{A}\right) \cdot{ }^{*} \boldsymbol{\Sigma}_{1}^{A}, \\
\mathbf{D}_{22}^{\rho} & =\int_{x} \int_{y} \mathbf{E}_{B} \mathbf{R}_{B}^{T} \mathbf{X}_{B}^{T} \mathbf{X}_{B} \mathbf{R}_{B} \mathbf{E}_{B} d x d y \\
& =\left(\mathbf{R}_{B}^{T} \mathbf{J}_{B B}^{X} \mathbf{R}_{B}\right) \cdot{ }^{*} \boldsymbol{\Sigma}_{1}^{B}, \\
\mathbf{D}_{12}^{\rho} & =\mathbf{D}_{21}^{\rho T}=\int_{x} \int_{y} \mathbf{E}_{A} \mathbf{R}_{A}^{T} \mathbf{Y}_{A}^{T} \mathbf{X}_{B} \mathbf{R}_{B} \mathbf{E}_{B} d x d y \\
& =\left(\boldsymbol{\Sigma}_{2}^{B} \mathbf{R}_{B}\right) \cdot{ }^{*}\left(\mathbf{R}_{A}^{T} \boldsymbol{\Sigma}_{3}^{A T}\right) .
\end{aligned}
$$

In (B.2), the following definitions are used:

$$
\begin{aligned}
& \mathbf{K}_{A}=\operatorname{diagonal}\left[k_{x(1)}, k_{x(2)}, k_{x(3)}, \ldots, k_{x\left(2\left(N_{y}-1\right)\right)}\right] \text {, } \\
& \mathbf{K}_{B}=\operatorname{diagonal}\left[k_{y(1)}, k_{y(2)}, k_{y(3)}, \ldots, k_{y\left(2\left(N_{x}-1\right)\right)}\right] \text {, } \\
& \boldsymbol{\Sigma}_{1}^{A}=\int_{x} \operatorname{vect}\left(\mathbf{E}_{A}\right) \operatorname{vect}\left(\mathbf{E}_{A}\right)^{T} d x \\
& =\left[\Sigma_{1 i j}^{A}\right]_{2\left(N_{y}-1\right) \times 2\left(N_{y}-1\right)^{\prime}} \\
& \Sigma_{1}^{B}=\int_{y} \operatorname{vect}\left(\mathbf{E}_{B}\right) \operatorname{vect}\left(\mathbf{E}_{B}\right)^{T} d y \\
& =\left[\Sigma_{1 i j}^{B}\right]_{2\left(N_{x}-1\right) \times 2\left(N_{x}-1\right)}, \\
& \boldsymbol{\Sigma}_{2}^{B}=\int_{x} \operatorname{vect}\left(\mathbf{E}_{A}\right) \mathbf{X}_{B} d x=\left[\Sigma_{2 i j}^{B}\right]_{2\left(N_{y}-1\right) \times\left(N_{x}-1\right)}, \\
& \boldsymbol{\Sigma}_{3}^{A}=\int_{y} \operatorname{vect}\left(\mathbf{E}_{B}\right) \mathbf{Y}_{A} d y=\left[\Sigma_{3 i j}^{A}\right]_{2\left(N_{x}-1\right) \times\left(N_{y}-1\right)}, \\
& \boldsymbol{\Sigma}_{4}^{B}=\int_{x} \operatorname{vect}\left(\mathbf{E}_{A}\right) \frac{d \mathbf{X}_{B}}{d x} d x=\left[\Sigma_{4 i j}^{B}\right]_{2\left(N_{y}-1\right) \times\left(N_{x}-1\right)}, \\
& \Sigma_{5}^{A}=\int_{y} \operatorname{vect}\left(\mathbf{E}_{B}\right) \frac{d \mathbf{Y}_{A}}{d y} d y=\left[\Sigma_{5 i j}^{A}\right]_{2\left(N_{x}-1\right) \times\left(N_{y}-1\right)}, \\
& \mathbf{J}_{B B}^{X}=\int_{x} \mathbf{X}_{B}^{T} \mathbf{X}_{B} d x=\frac{1}{6} l_{x} \mathbf{C}_{2\left(N_{x}\right)}, \\
& \mathbf{J}_{A A}^{Y}=\int_{y} \mathbf{Y}_{A}^{T} \mathbf{Y}_{A} d y=\frac{1}{6} l_{y} \mathbf{C}_{2\left(N_{y}\right)}, \\
& \overline{\mathbf{J}}_{B B}^{X}=\int_{x} \frac{\partial \mathbf{X}_{B}^{T}}{\partial x} \frac{\partial \mathbf{X}_{B}}{\partial x} d x=l_{x}^{-1} \mathbf{C}_{1\left(N_{x}\right)}, \\
& \overline{\mathbf{J}}_{A A}^{Y}=\int_{y} \frac{\partial \mathbf{Y}_{A}^{T}}{\partial y} \frac{\partial \mathbf{Y}_{A}}{\partial y} d y=l_{y}^{-1} \mathbf{C}_{1\left(N_{y}\right)},
\end{aligned}
$$

$$
\begin{aligned}
& \Sigma_{1 i j}^{A}= \begin{cases}L_{x} e^{-(1 / 2) i\left(\bar{k}_{x(i)}+\bar{k}_{x(j)}\right) L_{x}} & \text { if } k_{x(i)}+k_{x(j)}=0 \\
-i\left(k_{x(i)}+k_{x(j)}\right)^{-1} & \\
\cdot e^{-(1 / 2) i\left(k_{x(i)}+\bar{x}_{x(i)}+k_{x(j)}+\bar{k}_{x(j)}\right) L_{x}} & \\
\cdot\left(e^{i\left(k_{x(i)}+k_{x(j)}\right) L_{x}}-1\right) & \text { if } k_{x(i)}+k_{x(j)} \neq 0,\end{cases} \\
& \Sigma_{1 i j}^{B}= \begin{cases}L_{y} e^{-(1 / 2) i\left(\bar{k}_{y(i)}+\bar{k}_{y(j)}\right) L_{y}} & \text { if } k_{y(i)}+k_{y(j)}=0 \\
-i\left(k_{y(i)}+k_{y(j)}\right)^{-1} & \\
\cdot e^{-(1 / 2) i\left(k_{y(i)}+\bar{k}_{y(i)}+k_{y(j)}+\bar{k}_{y(j)}\right) L_{y}} & \\
\cdot\left(e^{i\left(k_{y(i)}+k_{y(j)}\right) L_{y}}-1\right) & \text { if } k_{y(i)}+k_{y(j)} \neq 0,\end{cases} \\
& \Sigma_{2 i j}^{B}=-l_{x}^{-1} k_{x(i)}^{-2} \\
& \cdot e^{-(1 / 2) i\left(\bar{k}_{x(i)} L_{x}+2 k_{x(i)}\left(x_{j-1}+2 x_{j}+x_{j+1}\right)\right)} \\
& \cdot\left[e^{i k_{x(i)}\left(x_{j-1}+2 x_{j}\right)}-2 e^{i k_{x(i)}\left(x_{j-1}+x_{j}+x_{j+1}\right)}\right. \\
& +e^{i k_{x(i)}\left(2 x_{j}+x_{j+1}\right)}+i e^{i k_{x(i)}\left(x_{j-1}+x_{j}+x_{j+1}\right)} \\
& \left.\cdot k_{x(i)}\left(x_{j-1}-2 x_{j}+x_{j+1}\right)\right], \\
& \Sigma_{3 i j}^{A}=-l_{y}^{-1} k_{y(i)}^{-2} \\
& \cdot e^{-(1 / 2) i\left[\bar{k}_{y(i)} L_{y}+2 k_{y(i)}\left(y_{j-1}+2 y_{j}+y_{j+1}\right)\right]} \\
& \cdot\left[e^{i k_{y(i)}\left(y_{j-1}+2 y_{j}\right)}-2 e^{i k_{y(i)}\left(y_{j-1}+y_{j}+y_{j+1}\right)}\right. \\
& +e^{i k_{y(i)}\left(2 y_{j}+y_{j+1}\right)}+i e^{i k_{y(i)}\left(y_{j-1}+y_{j}+y_{j+1}\right)} \\
& \left.\cdot k_{y(i)}\left(y_{j-1}-2 y_{j}+y_{j+1}\right)\right], \\
& \Sigma_{4 i j}^{B}=-i l_{x}^{-1} k_{x(i)}^{-1} \\
& \cdot e^{-(1 / 2) i\left[\bar{k}_{x(i)} L_{x}+2 k_{x(i)}\left(x_{j-1}+2 x_{j}+x_{j+1}\right)\right]} \\
& \cdot\left[e^{i k_{x(i)}\left(x_{j-1}+2 x_{j}\right)}-2 e^{i k_{x(i)}\left(x_{j-1}+x_{j}+x_{j+1}\right)}\right. \\
& \left.+e^{i k_{x(i)}\left(2 x_{j}+x_{j+1}\right)}\right], \\
& \Sigma_{5 i j}^{A}=-i l_{y}^{-1} k_{y(i)}^{-1} \\
& \cdot e^{-(1 / 2) i\left(\bar{k}_{y(i)} L_{y}+2 k_{y(i)}\left(y_{j-1}+2 y_{j}+y_{j+1}\right)\right)} \\
& \cdot\left[e^{i k_{y(i)}\left(y_{j-1}+2 y_{j}\right)}-2 e^{i k_{y(i)}\left(y_{j-1}+y_{j}+y_{j+1}\right)}\right. \\
& \left.+e^{i k_{y(i)}\left(2 y_{j}+y_{j+1}\right)}\right] .
\end{aligned}
$$

In (B.2), the symbol $\left(.{ }^{*}\right)$ denotes the elementwise matrix multiplication defined in MATLAB [32] as follows:

$$
\text { A. }{ }^{*} \mathbf{B}=\mathbf{C} \text {, }
$$


where components of the output matrix $\mathbf{C}$ are determined by

$$
C_{i j}=A_{i j} \times B_{i j}
$$

Finally the symbol vect(•) in (B.3) denotes the transformation of a diagonal matrix $\mathbf{G}=$ diagonal $\left[g_{1}, g_{2}, g_{3}, \ldots, g_{n}\right]$ into the column vector defined by

$$
\operatorname{vect}(\mathbf{G})=\left\{g_{1}, g_{2}, g_{3}, \ldots, g_{n}\right\}^{T}
$$

\section{Finite Element Matrices: $K$ and $M$}

Consider

$$
\begin{aligned}
& \mathbf{K}= \frac{P}{6 l_{x} l_{y}} \\
& \cdot\left[\begin{array}{cccc}
2\left(l_{x}^{2}+l_{y}^{2}\right) & l_{x}^{2}-2 l_{y}^{2} & -\left(l_{x}^{2}+l_{y}^{2}\right) & -2 l_{x}^{2}+l_{y}^{2} \\
l_{x}^{2}-2 l_{y}^{2} & 2\left(l_{x}^{2}+l_{y}^{2}\right) & -2 l_{x}^{2}+l_{y}^{2} & -\left(l_{x}^{2}+l_{y}^{2}\right) \\
-\left(l_{x}^{2}+l_{y}^{2}\right) & -2 l_{x}^{2}+l_{y}^{2} & 2\left(l_{x}^{2}+l_{y}^{2}\right) & l_{x}^{2}-2 l_{y}^{2} \\
-2 l_{x}^{2}+l_{y}^{2} & -\left(l_{x}^{2}+l_{y}^{2}\right) & l_{x}^{2}-2 l_{y}^{2} & 2\left(l_{x}^{2}+l_{y}^{2}\right)
\end{array}\right], \\
& \mathbf{M}=\frac{\rho l_{x} l_{y}}{36}\left[\begin{array}{cccc}
4 & 2 & 1 & 2 \\
2 & 4 & 2 & 1 \\
1 & 2 & 4 & 2 \\
2 & 1 & 2 & 4
\end{array}\right]
\end{aligned}
$$

\section{Conflict of Interests}

The authors declare that there is no conflict of interests regarding the publication of this paper.

\section{Acknowledgments}

This research was supported by Boeing Company and in part by the National Research Foundation of Korea (NRF) funded by the Korean government (MSIP) (Grant no. NRF2012R1A2A2A01004482). The authors would like to appreciate the valuable advice of Dr. Jeong-Beom Ihn at Boeing Company.

\section{References}

[1] J. Rehder, P. Rombach, and O. Hansen, "Balanced membrane micromachined loudspeaker for hearing instrument application," Journal of Micromechanics and Microengineering, vol. 11, no. 4, pp. 334-338, 2001.

[2] S. C. Ko, Y. C. Kim, S. S. Lee, S. H. Choi, and S. R. Kim, "Micromachined piezoelectric membrane acoustic device," Sensors and Actuators A: Physical, vol. 103, no. 1-2, pp. 130-134, 2003.

[3] X. Yang, C. Grosjean, Y.-C. Tai, and C.-M. Ho, "A MEMS thermopneumatic silicone rubber membrane valve," Sensors and Actuators A, vol. 64, no. 1, pp. 101-108, 1998.

[4] K. Sato, "Forced vibration analysis of a composite rectangular membrane consisting of strips," Journal of Sound and Vibration, vol. 63, no. 3, pp. 411-417, 1979.

[5] J. W. S. Rayleigh and R. B. Lindsay, The Theory of Sound, Dover, New York, NY, USA, 1945.
[6] L. E. Kinsler, A. R. Frey, A. B. Coppens, and J. V. Sanders, Fundamentals of Acoustics, John Wiley \& Sons, New York, NY, USA, 1982.

[7] L. Bauer and E. L. Reiss, "Free vibrations of rhombic plates and membranes," The Journal of the Acoustical Society of America, vol. 54, no. 5, pp. 1373-1375, 1973.

[8] J. A. Masad, "Free vibrations of a non-homogeneous rectangular membrane," Journal of Sound and Vibration, vol. 195, no. 4, pp. 674-678, 1996.

[9] Y. Y. Kim and J. H. Kang, "Free vibration analysis of membranes using wave-type base functions," The Journal of the Acoustical Society of America, vol. 99, no. 5, pp. 2938-2946, 1996.

[10] G. W. Wei, "Vibration analysis by discrete singular convolution," Journal of Sound and Vibration, vol. 244, no. 3, pp. 535-553, 2001.

[11] H. Ersoy, Ö. Civalekb, and L. Özpolatb, "Free vibration analysis of rectangular membranes with variable density using the discrete singular convolution approach," Asian Journal of Civil Engineering, vol. 11, no. 1, pp. 83-94, 2010.

[12] V. H. Cortinez and P. A. A. Laura, "Vibrations of nonhomogeneous rectangular membranes," Journal of Sound and Vibration, vol. 156, no. 2, pp. 217-225, 1992.

[13] P. A. A. Laura, R. E. Rossi, and R. H. Gutierrez, "The fundamental frequency of non-homogeneous rectangular membranes," Journal of Sound and Vibration, vol. 204, no. 2, pp. 373-376, 1997.

[14] S. H. Ho and C. K. Chen, "Free vibration analysis of nonhomogeneous rectangular membranes using a hybrid method," Journal of Sound and Vibration, vol. 233, no. 3, pp. 547-555, 2000.

[15] W. X. Wu, C. Shu, and C. M. Wang, "Vibration analysis of arbitrarily shaped membranes using local radial basis functionbased differential quadrature method," Journal of Sound and Vibration, vol. 306, no. 1-2, pp. 252-270, 2007.

[16] P. Amore, "A new method for studying the vibration of nonhomogeneous membranes," Journal of Sound and Vibration, vol. 321, no. 1-2, pp. 104-114, 2009.

[17] S. Durvasula, "Natural frequencies and modes of skew membranes," The Journal of the Acoustical Society of America, vol. 44, no. 6, pp. 1636-1646, 1968.

[18] P. A. A. Laura, L. Ercoli, R. O. Grossi, K. Nagaya, and G. S. Sarmiento, "Transverse vibrations of composite membranes of arbitrary boundary shape," Journal of Sound and Vibration, vol. 101, no. 3, pp. 299-306, 1985.

[19] C. Zhao and G. P. Steven, "An asymptotic formula for correcting finite element predicted natural frequencies of membrane vibration problems," Communications in Numerical Methods in Engineering, vol. 12, no. 1, pp. 63-73, 1996.

[20] M. G. Milsted and J. R. Hutchinson, "Use of trigonometric terms in the finite element method with application to vibrating membranes," Journal of Sound and Vibration, vol. 32, no. 3, pp. 327-346, 1974.

[21] W. H. Chen and C. W. Wu, "Adaptable spline element for membrane vibration analysis," International Journal for Numerical Methods in Engineering, vol. 39, no. 14, pp. 2457-2476, 1996.

[22] A. Houmat, "Hierarchical finite element analysis of the vibration of membranes," Journal of Sound and Vibration, vol. 201, no. 4, pp. 465-472, 1997.

[23] A. Y. T. Leung, B. Zhu, J. Zheng, and H. Yang, "A trapezoidal Fourier p-element for membrane vibrations," Thin-Walled Structures, vol. 41, no. 5, pp. 479-491, 2003. 
[24] A. Houmat, "Free vibration analysis of arbitrarily shaped membranes using the trigonometric p-version of the finite-element method," Thin-Walled Structures, vol. 44, no. 9, pp. 943-951, 2006.

[25] U. Lee, Spectral Element Method in Structural Dynamics, John Wiley \& Sons, Singapore, 2009.

[26] U. Lee and J. Lee, "Spectral-element method for Levy-type plates subject to dynamic loads," Journal of Engineering Mechanics, vol. 125, no. 2, pp. 243-247, 1999.

[27] A. Chakraborty and S. Gopalakrishnan, "A spectrally formulated plate element for wave propagation analysis in anisotropic material," Computer Methods in Applied Mechanics and Engineering, vol. 194, no. 42-44, pp. 4425-4446, 2005.

[28] F. Birgersson, S. Finnveden, and C.-M. Nilsson, "A spectral super element for modelling of plate vibration. Part 1: general theory," Journal of Sound and Vibration, vol. 287, no. 1-2, pp. 297314, 2005.

[29] D. E. Newland, Random Vibrations, Spectral and Wavelet Analysis, Longman, New York, NY, USA, 1993.

[30] W. C. William, Solution of Partial Differential Equations, Lecture Note, Stanford University, Stanford, Calif, USA, 1981.

[31] W. H. Wittrick and F. W. Williams, "A general algorithm for computing natural frequencies of elastic structures," The Quarterly Journal of Mechanics and Applied Mathematics, vol. 24, no. 3, pp. 263-284, 1971.

[32] Matlab User's Guide, The MathWorks, Natick, Mass, USA, 1993. 


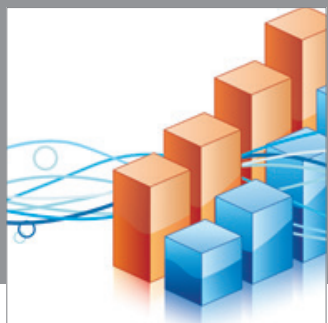

Advances in

Operations Research

mansans

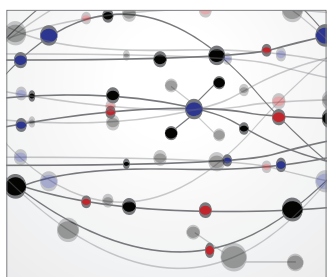

The Scientific World Journal
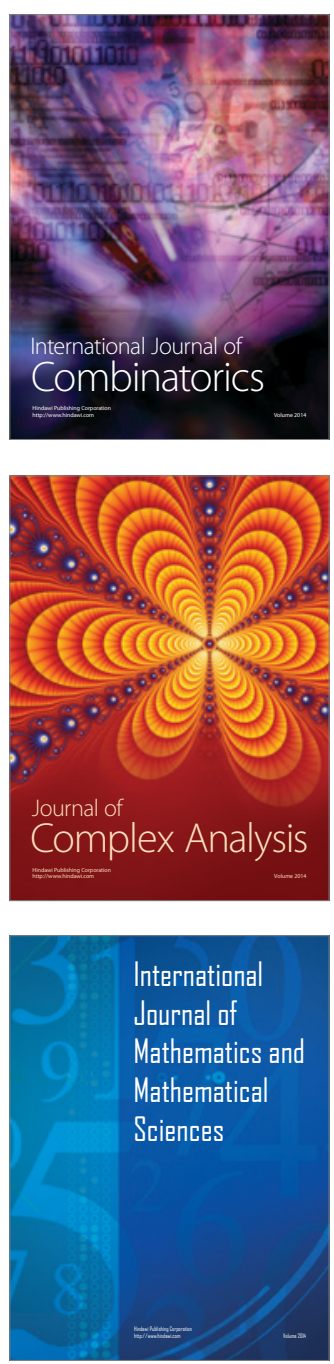
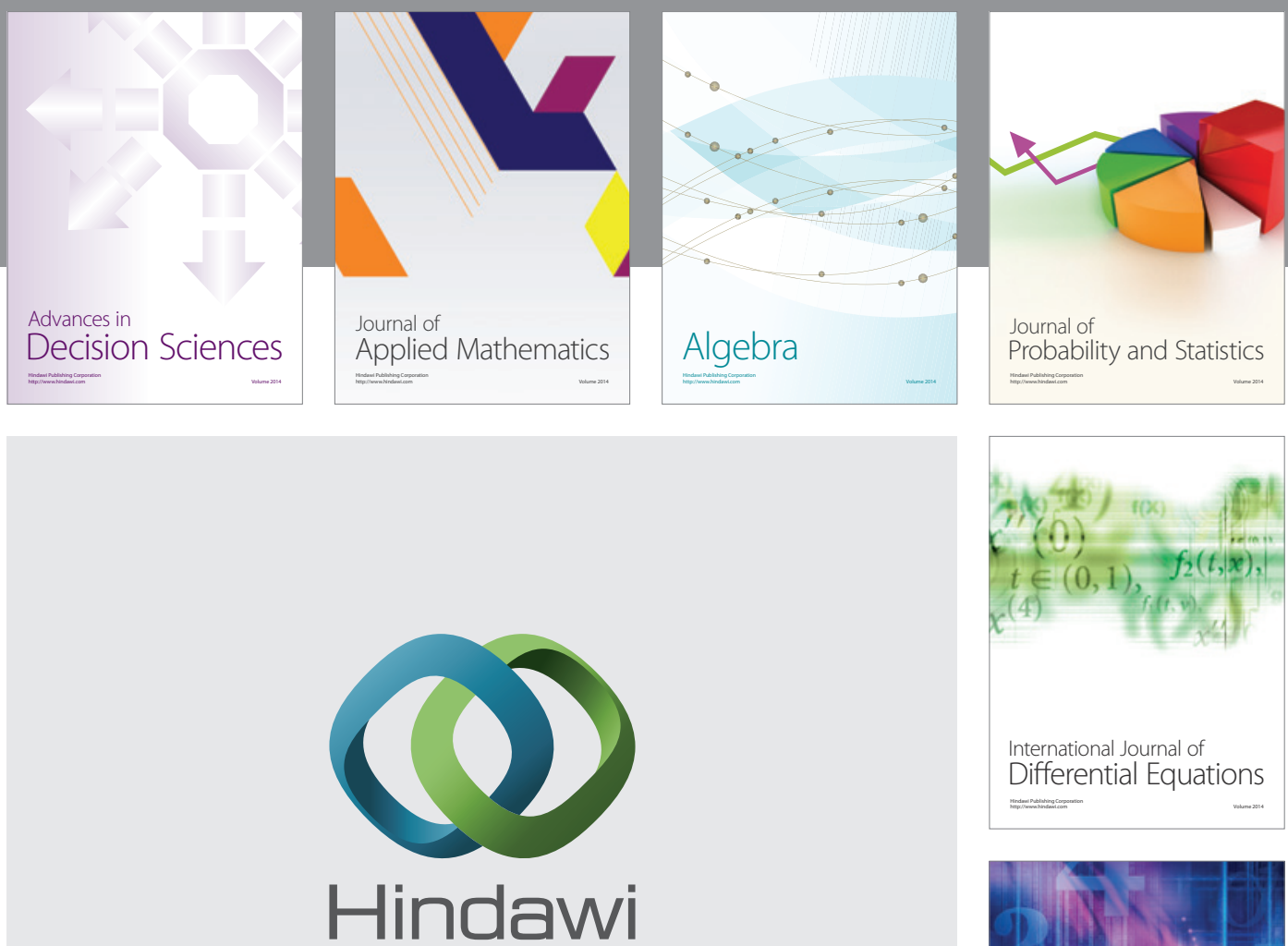

Submit your manuscripts at http://www.hindawi.com
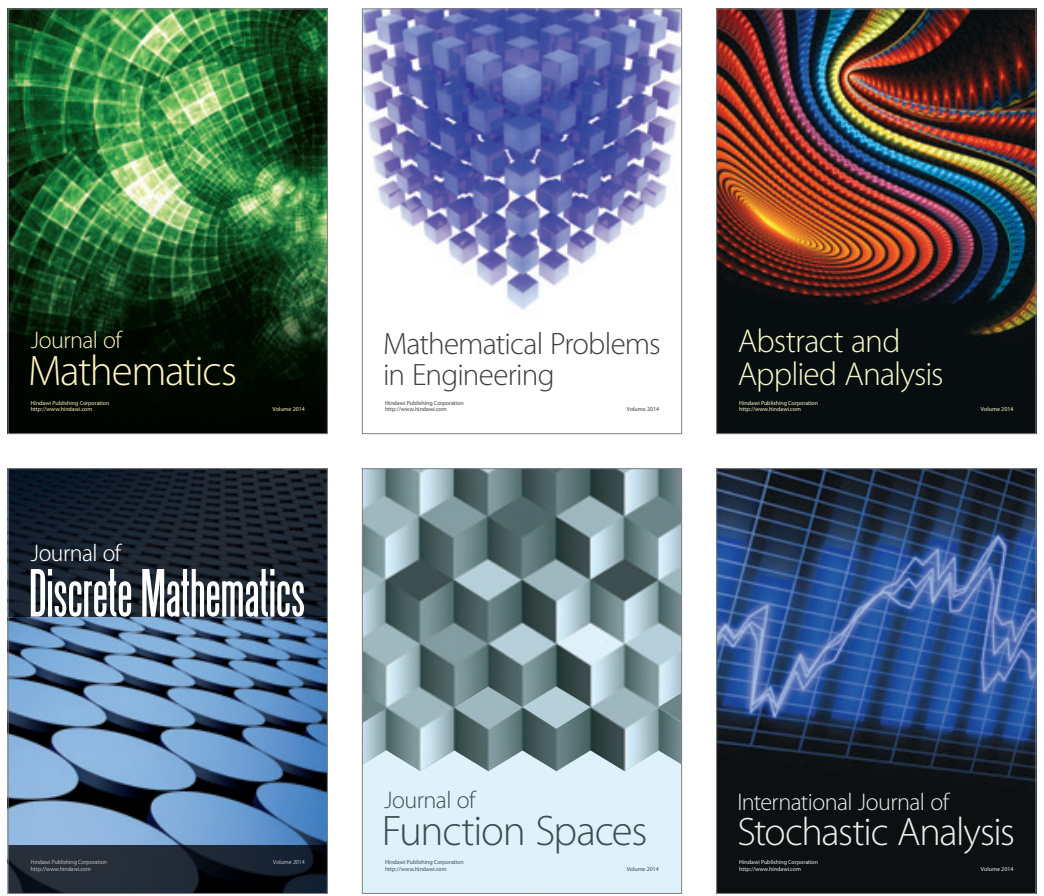

Journal of

Function Spaces

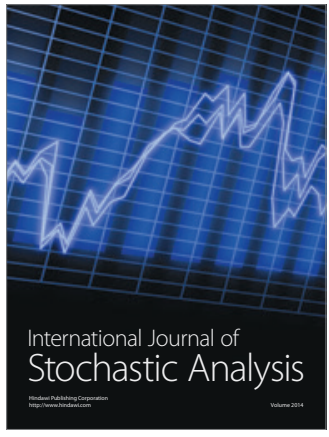

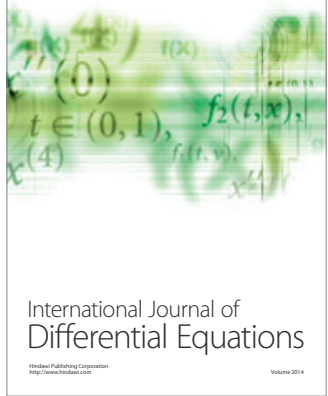
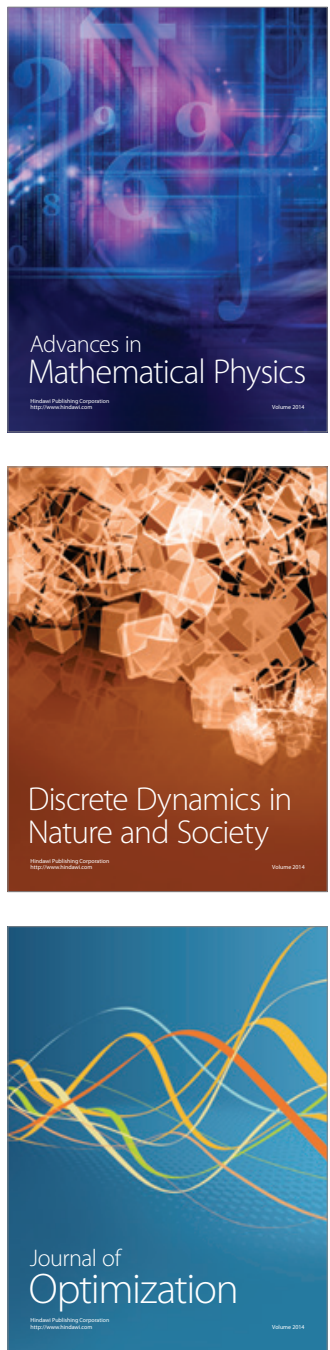